Cinémas

Revue d'études cinématographiques

Journal of Film Studies

\title{
Le genre co-opérateur
}

\section{André Gardies}

Volume 4, numéro 3, printemps 1994

Questions sur l'éthique au cinéma

URI : https://id.erudit.org/iderudit/1001036ar

DOI : https://doi.org/10.7202/1001036ar

Aller au sommaire du numéro

Éditeur(s)

Cinémas

ISSN

1181-6945 (imprimé)

1705-6500 (numérique)

Découvrir la revue

Citer cet article

Gardies, A. (1994). Le genre co-opérateur. Cinémas, 4(3), 43-55.

https://doi.org/10.7202/1001036ar

\section{Résumé de l'article}

Bien que relevant traditionnellement de la rhétorique, le gente est fondamentalement un acte pragmatique puisqu'il invite le spectateur à adopter le bon régime coopératif de lecture. Cependant, du genre fictionnel au genre factuel, quelque chose d'autre intervient : les valeurs de vérité du discours y fonctionnent de façon bien différente. En ce sens, en ce qui a trait à la fiction et au factuel, il sera préférable de parler de mode. L'une et l'autre sont au coeur de la question de l'éthique puisqu'ils engagent le statut de vérité de l'image et du discours audiovisuel. 


\title{
Le genre co-opérateur
}

\section{André Gardies}

\section{RÉSUMÉ}

Bien que relevant traditionnellement de la rhétorique, le genre est fondamentalement un acte pragmatique puisqu'il invite le spectateur à adopter le bon régime coopératif de lecture. Cependant, du genre fictionnel au genre factuel, quelque chose d'autre intervient: les valeurs de vérité du discours y fonctionnent de façon bien différente. En ce sens, en ce qui a trait à la fiction et au factuel, il sera préférable de parler de mode. L'une et l'autre sont au cœur de la question de l'éthique puisqu'ils engagent le statut de vérité de l'image et du discours audiovisuel.

\begin{abstract}
Though traditionnaly considered part of rhetoric, genre is fundamentally a pragmatic act because it invites the spectator to adopt the correct cooperative reading regime. However, between fictional and factual genres another consideration intervenes: the thruth values of discourse function in quite different ways. For this reason it seems preferable to speak of the fictional and the factual as modes. Both deal essentially with the question of ethics, since they entail the truth status of the image and of audiovisual discourse.
\end{abstract}

"Dans l'espace de la téléréalité, on ne sait plus où passe la frontière qui sépare le fantasme du vécu, le faux du vrai, le spectateur de l'acteur": cette affirmation d'origine journalistique ${ }^{\prime}$ résume assez bien, me semble-t-il, l'idée fort répandue suivant laquelle la "civilisation du live" engendrerait une confusion généralisée chez le téléspectateur. Il lui serait de plus en plus 
difficile de départager le réel et l'imaginaire, le faux et le vrai. D'autre part, la guerre du Golfe tout comme, pour me situer dans l'actualité française, l'affaire "B. H.", le meurtre de René Bousquet ou encore le suicide de Bérégovoy sont là pour nous rappeler que la télévision n'est plus seulement une merveilleuse et innocente "fenêtre ouverte sur le monde", qu'elle n'a plus seulement pour rôle de montrer la réalité, mais qu'elle peut aussi, par un effet boomerang, intervenir sur le réel en gauchissant des événements qui changent l'état du monde.

En conséquence se trouve posée, une fois de plus, la question du statut et du pouvoir de l'image, avec probablement plus d'acuité encore que par le passé récent. Avec plus de complexité aussi.

Car, à l'évidence, il ne s'agit plus d'interroger seulement l'image et son pouvoir de leurre, mais aussi le médium et le dispositif que celui-ci implique; c'est là, au moins, l'un des enseignements que l'on peut tirer de l'inflation télévisuelle. Une troisième donnée encore, jusque-là assez peu considérée (du moins dans la perspective de l'éthique audiovisuelle), doit être réévaluée, celle qui concerne la forme discursive, car ce ne sont pas seulement des images (et des sons) que produit le médium télévisuel, mais des images et des sons organisés en discours. Ce sont eux que le téléspectateur cherche à repérer en lisant la grille des programmes.

Statut ontologique de l'image, force illocutoire du médium, formes du discours audiovisuel, tels sont donc les trois lieux où se joue la question de l'éthique.

Mais, dira-t-on, ces remarques concernent la télévision et non le cinéma. Rien n'est moins sûr: l'ère de l'image électronique bouleverse la totalité du champ audiovisuel. Aux plans sociologique, esthétique et idéologique, le fait cinématographique ne peut plus être considéré comme un phénomène isolé, articulé qu'il est sur la totalité audiovisuelle ${ }^{2}$. Mélange d'images photographiques et numériques, "colorisation" du noir et blanc, contraintes du format, coproductions T.V. / cinéma, gauchissements scénaristiques et esthétiques imposés par la perspective d'une diffusion télévisuelle, etc. sont déjà quelques-uns des signes visibles de ces influences qu'il subit. Aussi, la question de l'éthique ne peut-elle plus se poser au niveau du cinéma seul; elle doit 
embrasser le champ de l'audiovisuel dans son ensemble tout en visant à "re-localiser" la spécificité cinématographique.

Vaste programme qui appelle un fil d'Ariane pour être parcouru. Aussi centrerai-je la réflexion sur les formes discursives et leur rapport au genre. En effet, c'est d'abord à des discours d'images et de sons que le (télé)spectateur a affaire: films de fiction, feuilletons, séries, dramatiques, documents, reportages, émissions de variétés, campagnes publicitaires aussi bien que politiques, reconstitutions en tous genres, journal télévisé, etc., rien ne semble devoir endiguer le déferlement des formes. Par contrecoup, le genre, en tant que forme-type reconnaissable et attendue par le spectateur - et donc susceptible de "canaliser" sa lecture - , perd peut-être son pouvoir de discrimination. Le régime de confusion généralisée, devenu fait de société, ne trouverait-il pas là une de ses causes essentielles?

\section{Le genre: un acte pragmatique}

Traditionnellement, le genre relève de la rhétorique: c'est elle, depuis des siècles, qui l'interroge et tente d'en fixer les règles, principes et canons. Or, du point de vue de sa fonctionnalité, particulièrement si l'on se place du côté du lecteur-spectateur, le genre est fondamentalement un acte pragmatique. N'est-ce pas, du reste, ce qui explique son retour dans le champ théorique aujourd'hui ${ }^{3}$ ?

En effet, dès lors qu'il se réfere, sous la diversité et la singularité des textes artistiques, à ce qui relève d'une sorte de forme type idéale (ayant valeur, lorsque la réflexion esthétique se fait normative, de modèle et de pierre de touche), le genre est tout à la fois relativement fixe et éminemment reconnaissable. Pour cette raison, il crée chez le lecteur-spectateur un horizon d'attente et, par là, conditionne son attitude coopérative. Essentiellement, le genre fonctionne comme régulateur dans l'acte d'appropriation du texte par le lecteur-spectateur. Opérateur du pacte de lecture, sa fonction (quelle que soit par ailleurs sa visée esthétique) est bien d'ordre pragmatique. En ce sens, il participe pleinement d'une éthique de la réception.

Avec lui, d'abord, la singularité d'un texte ne peut se saisir que dans la relation que celui-ci entretient avec les autres; et cela selon deux voies principales: l'histoire et l'intertextualité. 
Pour faire référence à un exemple fort connu, on sait qu' $I l$ était une fois dans l'Ouest, de Sergio Leone, doit une large part de son succès au rapport particulier qu'il institue avec le western. Tout à la fois, il répond aux données du genre et marque son originalité par un jeu d'écarts. Dès lors, il s'inscrit dans l'histoire cinématographique du western et je ne puis le lire sans tenir compte de ce passé sans lequel il ne saurait exister. Par ailleurs, tout spectateur attentif et amateur du genre ne manquera pas de repérer sous forme d'analogies, de similitudes ou de franches citations quelques westerns auxquels Il était une fois dans l'Ouest fait allusion, sinon référence.

La valeur pragmatique du genre apparaît ici dans une sorte de dimension collective : il articule le singulier (tel ou tel film particulier) sur un ensemble largement englobant. Dès lors, c'est moins au spectateur individuel qu'il s'adresse qu'à une sorte de communauté publique, celle qui justement fréquente ce genre. Et l'on sait combien sont vivaces (et changeants) les phénomènes de groupes adorateurs. Les affinités électives se reconnaissent dans la passion partagée d'un genre prisé en commun. Aimer un film au caractère générique clairement affiché, c'est tout à la fois aimer ce film et sa famille. Aimer par exemple Le Faucon maltais, c'est tout autant aimer cette histoire de ruses et de trahisons que Bogart, Joel Cairo, les pavés mouillés de la grande cité ou encore cette nébuleuse nocturne des films noirs américains.

Certes, sur la base de cette appartenance du singulier au générique, des jugements de valeur pourront être formulés, des hiérarchies pourront s'établir par comparaison - c'est la fonction esthétique qui entre en jeu -, mais antérieurement à cela, le genre aura fonctionné comme une sorte de protocole de lecture collectif. Il me rappelle que je ne saurais lire tel film singulier sans le situer au sein d'un vaste ensemble de règles, d'usages et de conventions, sans l'inscrire au sein d'une Histoire particulière, sans être sensible à la présence allusive d'autres films de la famille.

Cette nécessaire réinscription dans l'Histoire et l'intertextualité fonctionne alors comme une sorte de postulat narratif global: ce sont les données antérieures à la lecture du texte (chaque lecteur n'étant cependant pas obligé de les actualiser toutes) sur 
la base desquelles je vais élaborer un monde possible de références, nécessaire à l'évaluation des valeurs de vérité du discours. Manifestement, la vraisemblance des événements et des mésaventures propres au burlesque tient à ce que j'évalue la "vérité" de ces événements non par rapport au monde quotidien, de l'expérience vécue, mais par rapport à un monde imaginaire dont les lois different largement de celles qui gèrent ma réalité de chaque jour.

C'est précisément ma reconnaissance du genre "burlesque" qui me permet de trouver la bonne position coopérative ${ }^{4}$; et ceci pour mon plus grand plaisir puisque c'est à ce prix que je pourrai tirer profit du contrat comique et donc rire. La classique poursuite automobile, au milieu du trafic urbain, ne se conforme pas au même protocole selon que Harry Langdon se trouve au volant ou qu'il s'agit des protagonistes de Duel, par exemple; appliquer aux deux modes narratifs la même table de vérité serait évidemment un non-sens coopératif.

On comprend alors que l'industrie cinématographique ait très tôt, quitte à emprunter largement au fond artistique commun, développé et multiplié les genres. En indiquant au spectateur, à l'aide de marqueurs vite repérés et "appris", à quel genre de film il avait affaire, elle lui permettait d'adopter la bonne position coopérative et, par là, d'éviter les méprises dans le régime de lecture et donc les déceptions, sources de préjudice économique (sans compter que cette conformité à un modèle de référence autorisait une production à moindre coût ainsi que le montre l'existence des séries B). Le genre, au cinéma, est probablement plus de nécessité économique qu'esthétique. Cela explique sa rapide et étonnante extension eu égard à ce qu'a connu la littérature par exemple.

\section{Factuel / fictionnel}

Cela explique aussi probablement qu'une sorte de tabou ait été rarement transgressé: celui qui maintient une séparation nette entre le genre fictionnel et le genre documentaire (ou, pour se référer à un terme venu de la théorie du discours, le genre factuel). Le risque de déception pour le spectateur serait trop grand. Il semble bien, en effet, au regard de l'expérience 
commune, qu'il soit plus facile de s'accommoder de diverses transgressions concernant les "codes" du western par exemple, que d'une incertitude quant à la "vérité" des images qui me sont proposées.

C'est qu'en réalité la nature des transgressions n'y est pas du même ordre. Dans un cas, elle peut être source d'invention (au sein du genre fictionnel, la transgression d'aujourd'hui n'est bien souvent que la règle de demain), dans l'autre, elle met le statut même de l'image en question. Au reste, il n'est pas sûr que le terme de "genre" soit le mieux approprié pour désigner ces deux vastes ensembles que sont la fiction d'une part, le factuel de l'autre. Tout comme Gérard Genette 5 propose d'appeler "mode" les trois grands "genres" répertoriés par la rhétorique classique (le lyrique, le dramatique et l'épique), il serait plus judicieux de parler de mode fictionnel et de mode factuel, dont les genres constitueraient alors des sortes de sous-ensembles: la comédie musicale, le western, le film policier, le film érotique, le film d'aventures, etc. pour le premier; le reportage, le documentaire, l'enquête, les interviews, les actualités, etc. pour le second.

Pareil choix n'obéit pas seulement à quelque souci maniaque de classification, il répond à la nécessité de spécifier l'un des lieux où se joue la question de l'éthique. En effet, du mode fictionnel au mode factuel, c'est le statut du discours audiovisuel et sa valeur de vérité qui sont engagés.

Sans entrer dans le développement d'une question particulièrement complexe ${ }^{6}$, retenons une caractéristique essentielle. Le film documentaire tout comme le reportage et les genres du mode factuel sont censés avoir pour référent le monde réel de l'expérience vécue (selon une formule plus simple: ils nous parlent du monde où nous vivons). Cela a pour conséquence directe que la vérité de ce qu'ils nous disent a pour pierre de touche le monde réel. Mentir dans un film factuel, c'est mentir sur le monde.

Avec le film de fiction, il en va différemment. La vérité des propositions narratives qu'il contient s'évalue non par rapport au monde réel, mais par rapport au monde possible que le spectateur construit à partir des postulats narratifs propres à chaque film. Tout "mensonge" est un mensonge sur le film, non sur le 
monde réel. Hormis le cas toujours possible de la parodie, l'éventuelle présence dans un western (pour prendre un exemple inventé et grossier) d'une antenne de télévision serait "mensongère" par référence au monde postulé par le genre. Ce dernier suppose un certain état du monde, placé sous le signe de la civilisation du cheval, incompatible donc, eu égard à ma connaissance de ce monde, avec la présence d'une antenne de télévision.

En examinant plus attentivement ce qui est en jeu derrière cette distinction radicale entre le factuel et le fictionnel, on voit poindre la question fondamentale de la Vérité de l'image et du discours audiovisuel. En effet, que se passe-t-il lorsqu'un film me met en présence d'un fait inconnu et donc nouveau? Comment vais-je traiter cette information?

$\mathrm{Si}$ je postule (compte tenu des divers marqueurs intra ou " extratextuels") que je suis en régime fictionnel, j'ai le choix entre considérer ce fait nouveau comme une erreur ou comme l'indice d'un autre monde possible. Pour reprendre l'exemple de l'antenne de télévision, je peux soit décider qu'il s'agit d'une erreur de script (et dans ce cas, c'est la responsabilité du réalisateur qui est en cause), soit considérer que cette présence est juste. L'antenne devient alors un objet "vrai" d'un autre monde possible que je dois construire (rien n'empêcherait d'imaginer, par exemple, un village reculé de l'Ouest américain qui continuerait de vivre selon les lois du siècle précédent, malgré ici et là quelques apports du monde contemporain). C'est alors mon activité coopérative qui est en jeu, et non le travail du réalisateur.

Inversement, face à un film que je suppose être sur le mode factuel, l'élément nouveau sera traité différemment. Imaginons que dans un documentaire sur la vie sous-marine abyssale, on me montre un crustacé géant, de forme totalement inconnue, sans organes visuels mais avec des appendices tactiles démesurés, et que le commentaire me précise que cette espèce, particulièrement bien adaptée aux grandes profondeurs marines, vient d'être découverte, comment puis-je traiter cette information? Je n'ai pas d'autre choix que de la prendre comme vraie ou comme fausse. En effet, dès lors que le monde de référence du film est le monde physique réel, celui de l'expérience humaine, je ne peux pas le changer, je ne peux que modifier la connaissance que j'en 
ai (je ne peux donc pas construire un autre monde possible). Alors, soit je fais confiance à l'énonciateur du discours filmique (au besoin en prenant appui sur un principe de compatibilité: les caractéristiques énoncées du crustacé sont, par exemple, compatibles ou non avec les lois physiologico-physiques de son milieu marin telles que je les connais), soit je la considère comme mensongère. Dans le premier cas, l'image que j'ai du monde réel est modifiée (et le film m'apporte un savoir nouveau). Dans le second, c'est le discours audiovisuel que j'incrimine.

Du mode factuel au mode fictionnel, la question n'est pas de "genre", conçu comme ensemble de règles, codes et conventions construisant et régissant un monde possible, mais de statut ontologique du discours audiovisuel. C'est tout à la fois mon rapport au film et au monde réel qui est en jeu. C'est dire que la question de l'éthique s'y trouve posée frontalement.

\section{Le flux télévisuel}

Or ce que justement la "génération du live" peut brouiller, c'est cette frontière entre les deux modes. Et le dispositif télévisuel en est le lieu. En effet, l'institution cinématographique, elle, a besoin de bien marquer à la fois les genres et les modes (en témoignent les classifications par genres dans les hebdomadaires de programmes comme Pariscope ou L'Officiel des spectacles). Elle a besoin, pour sa survie économique, d'éviter les quiproquos avec ses spectateurs. Ces derniers doivent pouvoir trouver le bon régime coopératif sous peine éventuelle de déception ("éventuelle", car la déception momentanée - le "je n'ai rien compris ", pour parler de façon simpliste - peut toujours être retravaillée par le spectateur comme un nouvel apprentissage esthétique). Certes, il arrive que l'industrie cinématographique joue aux frontières des deux modes (que l'on songe, par exemple, aux documentaires animaliers de la firme Walt Disney et à leur dimension anthropomorphique), mais elle ne fait que jouer, sans pousser jusqu'à la transgression du tabou. Il n'en va pas de même avec le médium télévisuel.

Et cela parce que - la remarque en a été faite depuis longtemps - la télévision ne segmente pas, mais produit au 
contraire un flux continu. On sait qu'elle a horreur de l'interstice (souvenons-nous du petit train-rébus ou des "interludes" qui comblaient l'attente entre deux émissions): dès que débute la tranche des programmes quotidiens (dans l'hypothèse d'une non-diffusion 24/24 heures), rien ne doit en arrêter le mouvement jusqu'à l'heure de fin. Se succèdent et s'enchaînent alors les émissions et les discours audiovisuels les plus divers où le factuel et le fictionnel s'entrelacent en permanence, rendant leur démarcation bien difficile.

À cela s'ajoute l'autre fabuleux pouvoir du médium, celui par lequel il peut s'adresser à nous en direct, en temps réel, et souvent avec un accompagnement interactif. C'est là le fameux temps électronique dont parle Paul Virilio ${ }^{7}$, celui qui fait la spécificité de ce médium par rapport au cinéma. Nombreuses sont les études qui ont montré la part de charge émotionnelle que comporte le direct. En un sens, il "colle" au plus près au réel. Mais l'on sait aussi, particulièrement depuis les événements de Roumanie, combien douteuse peut être cette réalité. Et l'on a appris aussi qu'il était bien difficile d'établir la vérité des images.

Quel type de problème eut à résoudre alors le téléspectateur? Ayant postulé, sur la foi de l'insertion de ces images dans le journal télévisé, qu'il était en mode factuel, soit il admettait la vérité du discours audiovisuel (et son image du monde réel pouvait changer), soit il la mettait en doute; mais pour cela, encore fallait-il qu'il en eût les moyens! Il était bien évident que, pour le plus grand nombre de téléspectateurs, les images du charnier de Tsimisoara, par exemple, n'étaient pas incompatibles avec ce qu'il pouvait prêter d'atrocités aux régimes totalitaires, celui de Ceauscescu notamment. Dès lors, quelles raisons pouvaient le conduire à émettre un doute puisque, de surcroît, il n'avait à sa disposition aucun autre moyen de vérification?

Dans cette perspective, le flux télévisuel et le direct qu'autorise l'électronique apparaissent bien comme deux facteurs déterminants d'une nouvelle ontologie de l'image. Où sont les garants de sa vérité? Quelle attitude coopérative dois-je ou puis-je adopter?

Un déplacement fort important s'est produit: aux genres, chargés de me signifier le bon régime de lecture à adopter, s'est 
substituée "l'émission", notion relativement floue mais dont le téléspectateur fait empiriquement l'apprentissage. Avec tel type d'émission, il saura qu'il a affaire au mode factuel (le journal télévisé, les magazines, les variétés, le télé-achat, etc.) avec tel autre, il saura qu'il devra adopter plutôt le régime fictionnel (films, feuilletons, séries, etc.). Mais subsistent des zones incertaines: avec les pièces de théâtre, par exemple, où il oscille entre le sentiment d'être en présence de l'enregistrement d'un spectacle ("Au théâtre ce soir" en fut longtemps, en France, l'exemple canonique) et sa participation au monde diégétique de la pièce.

Bien entendu, avec la "génération du live", la difficulté s'est accrue, car le flottement a gagné le territoire du direct, atout majeur de la télévision. Être là au moment où l'événement se produit, cette règle de base du journalisme de reportage, rendue plus impérieuse encore depuis le développement de l'image électronique, devient un impératif spectaculaire. Là où la caméra n'aura pu être présente, rien n'empêchera de jouer à la reconstitution (il est vrai que les Lumière et Méliès, déjà, avaient ouvert cette voie). La mise en scène du réel (principe du "reality-show") joue fondamentalement sur l'ambivalence du mode discursif et sur l'indécision du téléspectateur quant au régime coopératif à adopter. Doit-il opter pour le mode factuel ou le mode fictionnel ? La frontière entre les deux s'est effacée. Le discours audiovisuel tout à la fois conjugue les événements reconstitués sur le mode hypothétique ("cela a dû se passer ainsi») et leur exposé sur le mode assertif du direct. Ni récit d'événements passés (mode narratif classique), ni prise directe sur les événements, la confusion devient la règle; il s'agit alors moins d'informer sur le monde (de modifier donc la connaissance que j'en ai) que de faire rêver sur le réel ("si j'avais été à la place de cet homme ou de cette femme, qu'aurais-je fait, comment aurais-je réagi ?").

L'industrie du rêve, dont le cinéma, a-t-on dit, fut longtemps le promoteur, s'est certes étendue à la télévision mais, ce faisant, elle a surtout changé de nature. Avec le film de fiction, je rêve, car je construis des mondes possibles dont je sais qu'ils sont pure fiction; avec le live, je rêve sur un réel fondamentalement irréel sans pouvoir, pour autant, le saisir comme fiction. Je dois le lire 
sur le mode factuel, sans pouvoir lui appliquer la règle de vérité propre à ce mode. C'est un réel pour rien, puisqu'il ne modifie en rien la connaissance que j'ai du réel. C'est du rêve, mais sans le pouvoir ludique de ce rêve qu'est la fiction.

Face à ce brouillage des modes, c'est l'institution télévisuelle qui sert de garant (avec tous les dangers que représente la mainmise ou le contrôle étatique sur cette institution): la grille des programmes et la nature annoncée de l'émission (c'est-à-dire un ensemble de critères externes au discours audiovisuel) deviennent les repères "coopératifs" du téléspectateur. Je sais qu’à 20 heures sur telle et telle chaîne, je serai en présence du mode factuel, par l'intermédiaire du journal télévisé, que tel soir de la semaine, entre 20 h 45 et 22 h 15, j'aurai soit un film, soit une émission de variétés, soit un grand magazine, etc. Je fais alors confiance à ces données externes, le plus souvent, du reste, incarnées par la figure du présentateur, responsable public de l'émission.

Alors une sorte de duplicité constitutive caractérise la télévision: elle manifeste un souci affiché de segmentation (la grille des programmes et le respect scrupuleux des horaires le disent assez clairement), dans le même temps que par le flux continu qui l'anime, elle ne cesse, en brassant divers types de discours, de rendre difficile la bonne perception des modes factuels et fictionnels. Et lorsqu'on sait qu'avec ces deux modes, c'est le statut de vérité de l'image qui est en jeu, on mesure mieux l'importance des enjeux.

Mais, à nouveau dira-t-on, qu'en est-il du cinéma là-dedans? N'est-ce pas l'affaire de la télévision seule? On sait par expérience déjà que ces deux "médias" sont trop proches (langagièrement et surtout économiquement) pour ne pas s'influencer réciproquement.

Certes, tant que l'industrie cinématographique a besoin des genres et des modes, on peut être assuré qu'elle évitera les brouillages "coopératifs", mais rien ne dit qu'il en sera toujours ainsi. J'en veux pour preuve cette contrainte économique et technologique qui fait que le tournage en vidéo l'emporte de plus en plus souvent sur le tournage en support film. Il ne s'agit pas seulement d'un changement de support, mais d'une transformation 
radicale: de l'image filmo-photographique, on passe à l'image électronique, avec les immenses possibilités de trucage qui sont les siennes. L'image ne pourra bientôt plus se porter garante de la présence dans le champ de l'objet filmé. La distinction " coopérative" ne s'établira plus entre un monde réel (fondement du film factuel) et un monde possible (fondement du film fictionnel), mais entre le monde réel et les mondes virtuels. Et ces derniers, en ressemblant de plus en plus étrangement, grâce au progrès technologique, au monde de notre expérience réelle, pourraient bien devenir indiscernables pour le spectateur. Dès lors la responsabilité du réalisateur sera plus fortement engagée encore.

Rien d'étonnant alors à ce qu'un cinéaste comme Chris Marker nous ait déjà prévenu du danger. Ambassade se présente comme un film d'amateur (l'absence de générique renforçant cet effet), témoignage direct de la vie quotidienne des réfugiés à l'ambassade de France au Chili, au lendemain du coup d'état de Pinochet. Toutes les caractéristiques du film (caméra tenue à la main, lumière imparfaite, cadrages incertains, commentaire rapporté après coup, etc.) sont là pour signifier la présence du cinéaste amateur parmi les réfugiés, lui-même partageant ce statut. C'est donc sous le signe de l'authenticité la plus complète que se présente ce court métrage. Or, les toutes dernières images, prises depuis la fenêtre de l'ambassade, montrent une rue plutôt européenne et enfin, au-dessus des toits, la tour Eiffel. À l'évidence, ce "document " est totalement reconstitué, il relève du "reality-show", mais il se désigne lui-même, de l'intérieur, comme "trucage", faisant ainsi surgir le véritable statut de son discours tout en désignant le leurre sur lequel il fonctionnait jusque-là. Par là, il redit au spectateur cette chose essentielle, qu'en matière d'images et de discours audiovisuel, on ne saurait jamais être assez vigilant.

Université Lumière-Lyon II

\section{NOTES}

1 Philippe Gavi, "Vivre et mourir en direct ", Le Nouvel Observateur, n 1493 (du 17 au 23 juin 1993). 
2 Serge Daney, notamment dans L'Exercice a été profitable, Monsieur (Paris: P.O.L., 1993), a bien marqué la réalité et l'importance de ce changement.

3 Sur ce point, le petit ouvrage, dense et efficace, de Dominique Combes, Les Genres littéraires (Paris: Hachette, 1992), est tout à fait explicite. Il montre bien comment, pour la période actuelle, le retour du genre s'oppose à la théorie du texte des années soixante-dix.

4 C'est naturellement Umberto Eco qui, en filigrane, se trouve ici convoqué, via notamment Lector in fabula (Paris: Grasset, 1985).

5 En particulier dans Fiction et diction (Paris: Seuil, 1991).

6 Sur ce problème, on pourra consulter notamment Univers de la fiction de Thomas Pavel (Paris: Seuil, 1988).

7 Notamment dans "Virilio aime la vidéo " dans Jean-Paul Fargier, Où va la vidéo? (Paris: Cahiers du cinéma, 1986), p. 25-31. 